\title{
Urinary Cotinine-Verified Smoking Status and Serum Lipid Profiles and Lipid Ratios in Korean Older Adults: A Nationwide Cross-Sectional Study
}

\section{Myung Ji Nam}

Korea University Ansan Hospital

\section{Chung-woo Lee}

Korea University Ansan Hospital

Hyunjin Kim

Korea University Ansan Hospital

Ji Won Yoo

University of Nevada Las Vegas School of Medicine

Kyu Na Lee

Soongsil University

Kyungdo Han

Soongsil University

Jin-Hyung Jung

Catholic University College of Medicine

\section{Yong-Gyu Park}

Catholic University College of Medicine

Do-Hoon Kim ( $\nabla$ kmcfm@hanmail.net)

Korea University Ansan Hospital

Joo-Hyun Park

Korea University Ansan Hospital

\section{Research Article}

Keywords: Smoking, Cotinine, Cholesterol, Dyslipidemia, Aged

Posted Date: December 21st, 2021

DOl: https://doi.org/10.21203/rs.3.rs-1186742/v1

License: (c) (i) This work is licensed under a Creative Commons Attribution 4.0 International License.

Read Full License 


\section{Abstract}

Background: Serum lipid profile is an important factor in predicting the risk of cardiovascular disease, and smoking is considered to affect serum lipid levels adversely by altering lipid metabolism. Although traditional lipid profiles are important in patient evaluation for predicting the risk of cardiovascular disease, the clinical significance of lipid ratios has recently gained attention as well. We aimed to investigate the relationship between smoking and serum lipid profiles and ratios in Korean older adults.

METHODS: This cross-sectional analysis used a nationally representative sample from the 2014-2016 Korea National Health and Nutrition Examination Survey (KNHANES). In total, 4,349 participants aged $\geq 60$ years with urine cotinine values were included. The outcome was a comparison of unfavorable lipid profiles/ratios in respect to urine cotinine-verified smoking status (urine cotinine level $\geq 50 \mathrm{ng} / \mathrm{mL}$ ), and quantification of the associations between smoking and unfavorable lipid profiles/ratios using multivariable logistic regression analyses. In our analyses, the lowest quartile (Q1) of high-density lipoprotein cholesterol (HDL-C), and the highest quartile (Q4) of low-density lipoprotein cholesterol (LDLC), triglycerides (TG), and total cholesterol (TC), and their ratios (TC/HDL-C, LDL-C/HDL-C, and TG/HDLC) were considered as unfavorable.

Results: The percentages of unfavorable lipid profiles/ratios except for serum TC and LDL-C were significantly higher in the urine cotinine-verified smoker group. The odds ratios (ORs) and $95 \%$ confidence intervals (Cls) of urine cotinine-verified smokers for the Q1 of HDL-C and for the Q4 of TG, TC/HDL-C, LDL-C/HDL-C, and TG/HDL-C were 1.67 (95\% Cl: 1.29-2.17) and 1.49 (95\% Cl: 1.14-1.96), 1.50 (95\% Cl: $1.14-1.97), 1.44$ (95\% Cl: $1.10-1.89)$, and 1.75 (95\% Cl: 1.35-2.28), respectively.

Conclusion: Urine cotinine-verified smoking status was significantly associated with unfavorable serum lipid profiles/ratios in Korean older adults.

\section{Background}

Dyslipidemia is an important factor in predicting cardiovascular risk, especially in older adults [1-4]. Abnormal serum lipid profiles, such as elevated low-density lipoprotein cholesterol (LDL-C), triglyceride (TG), and total cholesterol (TC) levels and decreased high-density lipoprotein cholesterol (HDL-C) levels, define dyslipidemia. In contrast, elevated HDL-C levels are considered protective against cardiovascular diseases [5].

Many studies have examined the effects of smoking on lipid metabolism. Although the mechanism is not yet comprehensively understood, several studies have found that smoking induced lipolysis and increased insulin resistance [6]. Other studies have attempted to describe the relationship between smoking and serum lipid profiles but have shown conflicting results [7-13]. Most of these studies were limited to Western populations and middle-aged people, who are more inclined and motivated to quit smoking. In addition, most of the studies focused mainly on traditional fasting plasma lipid profiles. 
Further, such studies also resorted heavily on self-reported questionnaires in assessing smoking status, which might have resulted in increased degree of biases.

Recent studies have shown that self-reporting leads to an underestimation of the prevalence of smoking compared to the use of biological sampling method [14]. Since nicotine has a shorter half-life (2 hours) than cotinine (12-15 hours), biological sampling of cotinine is more frequently used to evaluate smoking status. And urine sampling is a preferred method in biologic sampling of cotinine, due to non-invasive sample acquisition and higher concentrations of cotinine identification $[15,16]$. Therefore, we used urinary cotinine values to classify smokers and non-smokers, instead of relying on self-reported questionnaires. After reviewing past studies, we used a cutoff value of $50 \mathrm{ng} / \mathrm{mL}$ to differentiate urine cotinine-verified smokers from non-smokers in our study $[10,11,17]$.

Although traditional lipid profiles are still the cornerstone of patient evaluation, lipid ratios such as TC/HDL-C, TG/HDL-C, and LDL-C/HDL-C have recently gained attention for their predictive value for coronary heart disease $[18,19]$. For example, the high TC/HDL-C ratio may represent an atherogenic metabolic profile associated with insulin resistance and ischemic heart disease risk [20]. The TG/HDL-C ratio is also a predictor of insulin resistance [21] and is used to assess the size of the LDL-C particle. A high TG/HDL-C ratio indicates the presence of small LDL-C particles that are associated with a three-fold increase in the risk of myocardial infarction compared with normal-sized LDL-C particles [22]. A high LDL$\mathrm{C} / \mathrm{HDL}-\mathrm{C}$ ratio can predict sudden cardiac death risk better than individual lipid profiles and is associated with coronary plaque progression [23]. Despite these clinical significances, however, there has not been sufficient research on the relationship between smoking and lipid ratios.

Therefore, we aimed to evaluate the relationship between urine cotinine-verified smoking status and serum lipid profiles and their ratios in Korean older adults while adjusting for potential confounders. The study subjects' unfavorable lipid profiles and ratios were compared in respect to urine cotinine-verified smoking status. Additionally, the associations between urine cotinine-verified smoking status and unfavorable serum lipid profiles and their ratios were evaluated. Data from the Korea National Health and Nutrition Examination Survey (KNHANES) were used to increase the generalizability of our findings.

\section{Methods}

\section{Study participants}

We used datasets from the 2014-2016 KNHANES in this study. The KNHANES is a population-based, nationwide, cross-sectional survey conducted by the Korea Center for Disease Control and Prevention since 1998. The study participants were randomly selected using stratified, multistage-clustered probability sampling of the general Korean population. The survey database contains data on demographics and income status (including socioeconomic factors), nutrition, health screening records, and health interview questionnaires; well-trained research assistants perform the health interviews and dieticians administer the nutrition-related questionnaires. 
In the 2014-2016 KNHANES, urinary cotinine levels were measured in 16,054 participants. After selecting participants aged 60 years or older, 5,171 participants remained. We then excluded individuals who indicated that they had been diagnosed with various cancers or chronic liver disease to minimize the effect of underlying diseases on lipid profiles. In total, 4,349 participants were included in the final analysis (Figure 1). All data we used were downloaded from the KNHANES homepage (https://knhanes.kdca.go.kr) and were anonymized and de-personalized. The survey participants have provided written informed consent prior to the completion of the survey. The 2014 KNHANES was reviewed and approved by the Institutional Review Board (IRB) of the Korea Center for Disease Control and Prevention (IRB approval no. 2013-12EXP-03-5C) and IRB for the subsequent KNHANES was waived on the grounds of anonymity of the data.

\section{Measures}

Demographic variables such as age, level of education (highest level of education), and monthly household income (categorized into quartiles) of the participants were identified. The study subjects who were high school graduates or higher were categorized as the 'high education level' group in our study. The study subjects who were lowest $25 \%$ for the income according to the National Statistics data were categorized as 'low income' group in our study. Furthermore, data on lifestyle variables such as alcohol intake (percentage of individuals who drank more than once a month during the previous year), smoking status (percentage of current smokers), physical activity (percentage of those who exercised moderately for more than 2 hours and 30 minutes a day, who exercised vigorously for more than 1 hour and 15 minutes a day, or who performed an equivalent amount of mixed exercise), and daily energy intake, with daily carbohydrate, protein, and fat intake, were obtained. Daily energy and fat intake (the percentages of fat per total food consumed in 24 hours) were assessed using the 24-hour recall method in the nutritional survey portion of the KNHANES. The medical history, family history of dyslipidemia, and current medications of the participants were evaluated using a questionnaire.

Spot urine samples were obtained by instructing the survey participants to collect a midstream first morning void. Urine cotinine levels were analyzed using a gas chromatography-mass spectrometer detector (GC-MSD) Clarus 600/600T (Perkin Elmer, USA) in the 2014-2015 KNHANES. High-performance liquid chromatography with mass spectrometry (HPLC-MS/MS) using the Agilent 110 series with API 4000 (AB Sciex, USA) was used to quantify urine cotinine levels in the 2016 KNHANES. Based on previous studies, the study subjects with urine cotinine level of $<50 \mathrm{ng} / \mathrm{mL}$ were classified as non-smokers, and the study subjects with urine cotinine level $\geq 50 \mathrm{ng} / \mathrm{mL}$ were classified as smokers $[10,11,17]$.

Participants were measured for height and body weight only wearing light indoor clothing. Waist circumference was measured to the nearest $0.1 \mathrm{~cm}$ at the narrowest point between the costal margin and the iliac crest at the end of exhalation. Body weight $(\mathrm{kg})$ was divided by the square of height $\left(\mathrm{m}^{2}\right)$ to calculate body mass index (BMI). Blood pressure was measured three times at a right arm with each 
study subject in a seated position, following a five-minute long resting period; the average of the three measurements was calculated for the study subjects.

Blood samples were obtained after fasting for $\geq 8$ hours, from the antecubital vein. Samples were immediately refrigerated, transported under cold storage conditions to the Central Testing Institute in Seoul, Korea. The serum concentrations of serum lipids, TC, HDL-C, LDL-C, and TG, were measured using a Hitachi Automatic Analyzer 7600-210 (Hitachi, Tokyo, Japan) via enzymatic methods using commercially available kits (Sekisui, Japan). All samples were analyzed within 24 hours of transportation.

\section{Statistical analyses}

Descriptive analyses were conducted on the baseline characteristics of cotinine-verified smoking group (urinary cotinine level $\geq 50 \mathrm{ng} / \mathrm{mL}$ ) and non-smoking group (urinary cotinine level $<50 \mathrm{ng} / \mathrm{mL}$ ). The results were expressed as 'arithmetic mean \pm standard error (SE)' for the continuous variables except for urine cotinine and serum triglycerides, whose results were expressed as 'geometric mean ( $95 \%$ confidence intervals [Cls])' due to the skewness of their distributions; the results for the categorical variables were expressed in 'weighted percentage (SE).' The two groups' baseline characteristics were then compared using Student's t-tests (for continuous variables) and chi-square tests (for categorical variables).

The distributions of unfavorable quartiles (Q) of lipid profiles and ratios, such as the highest quartile (Q4) of TC, LDL-C, TG, TC/HDL-C, LDL-C/HDL-C, and TG/HDL-C and the lowest quartile (Q1) of HDL-C, were compared between the two groups using chi-squared tests. In addition, subgroup analyses by gender were conducted. Of note, the cut-offs that differentiated between Q1 vs. Q2-4 and Q4 vs. Q3-1 were generated in a gender stratified manner.

In order to quantify the association between urine cotinine-verified smoking status and unfavorable serum lipid profiles and ratios, multivariable logistic regression analyses were performed. In the logistic regression, the binary variables transformed from serum lipid profiles and ratios were used as the dependent variables. For serum TC, LDL-C, TC/HDL-C, LDL-C/HDL-C, and TG/HDL-C, the cut-off divided each variable into Q4 and the rest (Q3-1). For HDL-C, the cut-off divided the variable into Q1 and the rest (Q2-4).

The regression analyses used multiple levels of adjustment; model 1 was adjusted for age, sex, BMI, level of income, and level of education. Model 2 was further adjusted for alcohol consumption, physical activity, family history of dyslipidemia, DM, HTN, lipid-lowering drug use, energy intake, and fat intake. The analyses produced the odds ratios (ORs) and $95 \% \mathrm{Cls}$ of various unfavorable lipid profiles and ratios for urinary cotinine-verified smoking status. Additional analyses were conducted on male and female subgroups using the same method. All statistical analyses were performed using SAS version 9.3 software (SAS Institute, Cary, NC, USA). Statistical significance was set at two-sided $p$-values $<0.05$. 


\section{Results}

\section{Baseline characteristics of the participants}

Among the 4,349 study subjects, 593 were categorized as smokers (urine cotinine level $\geq 50 \mathrm{ng} / \mathrm{mL}$ ) and 3,756 as non-smokers (urine cotinine level $<50 \mathrm{ng} / \mathrm{mL}$ ). The geometric mean of urine cotinine was 699.2 $\mathrm{nmol} / \mathrm{L}$ for the cotinine-verified smokers and $0.8 \mathrm{nmol} / \mathrm{L}$ for the cotinine-verified non-smokers $(\mathrm{p}<$ $0.0001)$. The proportion of men were significantly greater among the cotinine-verified smokers $(81.8 \%)$ than the cotinine-verified non-smokers (38.4\%). The proportion of the study subjects who responded as having drunk more than once a month in the preceding year were significantly greater in the cotinineverified smoker group (59.1\%) than the cotinine-verified non-smoker group (36.8\%). No significant differences were found between the two groups in terms of the proportion of those who exercised regularly, those who reported a diagnosis of DM or HTN, and daily fat intake. However, significant differences existed in lipid-lowering drug use (smokers $12.4 \%$ vs. non-smokers $22.8 \%$ ) and daily energy intake (smokers 1,771.6 $\pm 35.1 \mathrm{kcal}$ vs. non-smokers 1,690.7 $\pm 14.7 \mathrm{kcal}$ ) between the two groups.

Among the serum lipid profiles and ratios, only LDL-C/HDL-C ratios of the two groups did not differ significantly $(p=0.121)$.

\section{The differences in the distributions of various unfavorable lipid profiles and ratios in respect to cotinine-verified smoking status}

Comparison of the distributions of unfavorable quartiles of lipid profiles between the cotinine-verified smokers and non-smokers is as shown in Figure 2. Gender-stratified comparison was also conducted. The proportion of the study subjects whose serum TC and LDL-C values were from the Q4 did not differ significantly between the cotinine-verified smoker group and the non-smoker group ( $26.94 \%$ vs. $25.58 \%$ and $25.12 \%$ vs. $25.63 \%$ ). However, the proportion of the study subjects whose serum TG values were from the Q4 and whose serum HDL-C values were from the Q1 were significantly higher in the cotinine-verified smoker group than in the non-smoker group ( $32.86 \%$ vs. $23.53 \%$ and $31.26 \%$ vs. $23.49 \%$ ).

Regarding lipid ratios, comparison of the distributions of unfavorable quartiles in respect to the cotinineverified smoking status showed that the proportion of the study subjects whose TC/HDL-C, LDL-C/HDL-C and TG/HDL-C ratios were from the Q4 were all significantly higher in the cotinine-verified smoker group than in the non-smoker group ( $31.94 \%$ vs. $24.14 \%, 29.71 \%$ vs. $24.2 \%$, and $33.84 \%$ vs. $23.47 \%$, respectively).

\section{Association of cotinine-verified smoking status with serum lipid levels and serum lipid ratios}


The ORs and $95 \%$ Cls of urine cotinine-verified smokers for various serum lipid profiles and ratios after adjustments with all the potential confounders are presented in Table 2. All of the cut-off values for the Response Variables are shown in Additional File 1

In the total population, the ORs $(95 \% \mathrm{Cl})$ of cotinine-verified smokers for Q1 of the HDL-C level was significantly increased to 1.67 (95\% Cl: 1.29-2.17), compared with Q2-Q4 values. The ORs of cotinineverified smokers were also significantly increased for Q4 of serum lipid levels and serum lipid ratios compared with Q1-Q3 values for each variable: TG, 1.49 (95\% Cl: 1.14-1.96); TC/HDL-C ratio, 1.50 (95\% Cl: 1.14-1.97); LDL-C/HDL-C ratio, 1.44 (95\% Cl: 1.10-1.89); and TG/HDL-C ratio, 1.75 (95\% Cl: 1.352.28). The ORs for Q4 of TC and LDL-C levels were not significant.

Of note, the ORs for lipid-lowering drug users (reference: non-users) in the total population were 0.21 (95\% Cl: 0.16-0.29) for TC Q4, 0.62 (95\% Cl: 0.49-0.80) for HDL-C Q1, 0.13 (95\% Cl: 0.09-0.18) for LDL-C Q4, 0.80 (95\% Cl: 0.65-0.98) for TG Q4, 0.20 (95\% Cl: 0.15-0.26) for TC/HDL-C Q4, 0.13 (95\% Cl: 0.10-0.18) for LDL-C /HDL-C Q4, and 0.71 (95\% Cl: 0.57-0.87) for TG/HDL-C Q4, respectively (work not shown in the table).

The Additional File 2 shows the results of the subgroup analyses. The male and female groups showed a similar trend in that the ORs for Q1 of the HDL-C level and Q4 of other serum lipid levels and lipid ratios. In the male group, the ORs for the cotinine-verified smokers were significantly increased in regard to Q1 of the HDL-C level (1.59, 95\% Cl: 1.17-2.15), and Q4 of the TG level, TC/HDL-C ratio, LDL-C/HDL-C ratio, and TG/HDL-C ratio (1.41, 95\% Cl: 1.03-1.93; 1.41, 95\% Cl: 1.04-1.92; 1.39, 95\% Cl: 1.03-1.89; 1.72, 95\% Cl: $1.29-2.29)$.

The findings were similar for the female group but were greater in magnitude. The ORs for the cotinineverified smokers were increased in regard to Q1 of the HDL-C level, Q4 of the TG level, TC/HDL-C ratio, and TG/HDL-C ratio. The values are as follows: $2.17(95 \% \mathrm{Cl}: 1.21-3.90), 1.77$ (95\% Cl: 1.03-3.04), 2.11 (95\% Cl: 1.16-3.84), and 1.89 (95\% Cl: 1.12-3.19), respectively.

\section{Discussion}

Our study found that cotinine-verified smoking status was significantly associated with unfavorable serum lipid profiles and ratios than non-smokers in Korean elderly population.

Our results were somewhat consistent with those of the several past studies. The study based on the 2008-2010 KNHANES showed increased ORs for high TG levels among cotinine-verified smokers in both female (OR, 1.45; 95\% Cl: 1.13-1.85) and male (OR, 1.30; 95\% Cl: 1.12-1.51) groups. The same study also reported a dose-dependent relationship between urine cotinine and TG ( $p$ for trend $<0.01$ for both men and women), as well as an inverse relationship between urine cotinine and HDL-C ( $p$ for trend $<0.01$ for men, 0.02 for women) [11]. According to another study conducted using the 2008-2010 KNHANES datasets, cotinine-verified smoking status showed a significant dose-dependent relationship with TG, HDL-C levels, and TC/HDL-C, LDL-C/HDL-C, and TG/HDL-C ratios [18]; however, this study was conducted 
only on Korean males In the cross-sectional study conducted in northwest rural China from 2008 to 2012 , the TC/HDL-C, TG/HDL-C, and LDL-C/HDL-C ratios were significantly higher in smokers, whereas HDL-C level was lower in smokers [8]; however, these results were mostly based on relatively "younger" adult participants who were aged 20 years or older.

There have also been a few prior studies that were conducted on "older" individuals. According to the 2011 study on 3,251 Chinese adults aged 45-89 years, smoking was associated with dyslipidemia (OR, $1.23 ; 95 \% \mathrm{Cl}: 1.01-1.51$ ) [7]. The study by Yasue et al. conducted from 1998 to 2001, with the mean age of $61.8 \pm 11.0$ years for the current smoker group and $68.2 \pm 10.7$ years for the never smoker group, concluded that HDL-C levels were significantly lower in the current-smoker group than in the never-smoker group $(45.2 \pm 12.3 \mathrm{mg} / \mathrm{dL}$ vs. $50.6 \pm 15.6 \mathrm{mg} / \mathrm{dL}, \mathrm{p}<0.01)$. However, that no significant difference was observed in TC levels between the two groups differed from our results [9].

As mentioned before, the mechanism by which smoking affects lipid metabolism is not fully understood; however, several previous studies have found that smoking increases lipolysis and insulin resistance. Nicotine stimulates the release of catecholamines that facilitate lipolysis and increase the concentration of plasma free fatty acids $[24,25]$. These catecholamines also antagonize the action of insulin, which would further impair insulin sensitivity. This adds to the effect of the hormones that oppose the function of insulin, such as glucagon, whose levels also increase in response to smoking $[25,26]$. Lipid levels or lipid oxidation states seem to be partially restored following smoking cessation, but more studies are needed to confirm this finding $[18,25]$. Further studies are also warranted to elucidate the key mechanisms involved in the relationship between smoking and dyslipidemia.

Our study had several limitations. First, as it was a cross-sectional study, it was difficult to derive causal associations between smoking and lipid profiles and ratios. We can only describe the direction and the strength of the associations between these variables. Second, although we classified smokers using urinary cotinine levels based on previous studies, individual genetic variations in factors such as CYP2A6, which encodes a protein involved in nicotine metabolism, could not have been accounted for [27]. Furthermore, although we used a reliable cutoff value, it was not feasible to differentiate between primary smokers and those who were exposed to secondhand cigarette smoke only based on the urinary cotinine levels that were available. Third, although our results were adjusted for energy and fat intakes, the effect of diet on serum lipid levels could not be adjusted in whole. Previous studies have shown that a low-fat diet resulted in higher ORs for low HDL-C levels [13] and was responsible for the reduction in serum LDL-C levels [28]. Fourth, since food intake was assessed using a 24-hour recall method in the KNHANES, the data used for the adjustment of energy and fat intake might be biased because due to individual differences in ability to recall. In addition, the data could not fully represent the participants' usual eating habits due to the intraindividual variations in daily food consumption.

Despite these limitations, our study is significant for several reasons. First, the study was conducted using a nationally representative data. Second, we adjusted for multiple potential confounders; the analyses were adjusted for age, sex, BMI, income level, education level, alcohol consumption, physical 
activity, family history of dyslipidemia, DM, HTN, use of lipid-lowering drugs, energy intake, and fat intake. Third, only a few studies have investigated a relationship between smoking and dyslipidemia profiles and their ratios in the older population. To the best of our knowledge, our study is the first to report the association between urine cotinine verified smoking status and serum lipids levels/ratios in older adult population. Older adults are only half likely as young smokers in attempting to quit smoking [29]. Smoking is a modifiable cardiovascular risk factor, and its cessation is beneficial even at an advanced age [30]. Our findings may further motivate older adults to consider reducing their cigarette smoking.

\section{Conclusions}

This nationwide study found that smoking was associated with unfavorable serum lipid profiles/ratios in elderly Korean individuals aged 60 years and older, which could then lead to an increased risk of cardiovascular diseases. As mentioned above, previous studies have found that smoking cessation could normalize, at least partially, the lipid profiles and eventually reduce relevant mortalities in older adults. Therefore, our study carefully suggests that smoking cessation could also be important among the old age as well. In the future, cohort studies that investigate on the changes in dyslipidemia parameters and serum lipid ratios in older adult population after smoking cessation should be conducted to verify the necessity and impact of smoking cessation in older adult population.

\section{Abbreviations}

LDL-C Low-density lipoprotein cholesterol

TG Triglycerides

TC Total cholesterol

HDL-C High-density lipoprotein cholesterol

DM Diabetes mellitus

HTN Hypertension

KNHANES Korea National Health and Nutrition Examination Survey

IRB Institutional Review Board

BMI Body mass index

SE Standard error

Cl Confidence intervals

OR Odds ratio 
Q Quartile

Q4 Highest quartile

Q1 Lowest quartile

\section{Declarations}

\section{- Ethics approval and consent to participate}

The 2014 KNHANES was reviewed and approved by the Institutional Review Board (IRB) of the Korea Center for Disease Control and Prevention (IRB approval no. 2013-12EXP-03-5C) and IRB for the subsequent KNHANES was waived on the grounds of anonymity of the data.

\section{- Consent for publication}

Not applicable

\section{- Availability of data and materials}

All dataset we used were downloaded from the KNHANES homepage (https://knhanes.kdca.go.kr) after submission and evaluation of an appropriate research proposal.

\section{- Competing interests}

The authors declare that they have no competing interests.

\section{- Funding}

This work was supported by a grant from the Korea University (K1912691). The funding body had no role in the study design, data collection and analysis, decision to publish, or preparation of the manuscript.

\section{- Authors' contributions}

All authors were responsible for study concept and design, analysis and interpretation of data, and critical revision of the manuscript for important intellectual content. Myung Ji Nam acquired the data and wrote the original draft. Do-Hoon Kim and Joo-Hyun Park were responsible for obtaining funding and study supervision. Chung-woo Lee, and Hyunjin Kim, and Ji Won Yoo discussed the results and commented on the manuscript. Kyu Na Lee, Kyungdo Han, Jin-Hyung Jung, and Yong-Gyu Park contributed to the 
statistical analysis. All authors have read and agreed to the published version of the manuscript. All the authors have agreed to accept equal responsibility for the accuracy and content of the paper.

\section{- Acknowledgments}

Not applicable

\section{References}

1. Castelli WP, Doyle JT, Gordon T, Hames CG, Hjortland MC, Hulley SB, et al. HDL cholesterol and other lipids in coronary heart disease. The cooperative lipoprotein phenotyping study. Circulation. 1977;55:767-72.

2. White J, Swerdlow DI, Preiss D, Fairhurst-Hunter Z, Keating BJ, Asselbergs FW, et al. Association of lipid fractions with risks for coronary artery disease and diabetes. JAMA Cardiol. 2016;1:692-9.

3. Hokanson JE, Austin MA. Plasma triglyceride level is a risk factor for cardiovascular disease independent of high-density lipoprotein cholesterol level: a meta-analysis of population-based prospective studies. J Cardiovasc Risk. 1996;3:213-9.

4. Zimetbaum P, Frishman WH, Ooi WL, Derman MP, Aronson M, Gidez LI, et al. Plasma lipids and lipoproteins and the incidence of cardiovascular disease in the very elderly. The Bronx Aging Study. Arterioscler Thromb. 1992;12:416-23.

5. Gordon T, Castelli WP, Hjortland MC, Kannel WB, Dawber TR. High density lipoprotein as a protective factor against coronary heart disease. The Framingham Study. Am J Med. 1977;62:707-14.

6. Gastaldelli A, Folli F, Maffei S. Impact of tobacco smoking on lipid metabolism, body weight and cardiometabolic risk. Curr Pharm Des. 2010;16:2526-30.

7. Wang S, Xu L, Jonas JB, You QS, Wang YX, Yang H. Prevalence and associated factors of dyslipidemia in the adult Chinese population. PLoS One. 2011;6:e17326.

8. Li XX, Zhao Y, Huang LX, Xu HX, Liu XY, Yang JJ, et al. Effects of smoking and alcohol consumption on lipid profile in male adults in northwest rural China. Public Health. 2018;157:7-13.

9. Yasue H, Hirai N, Mizuno Y, Harada E, Itoh T, Yoshimura M, et al. Low-grade inflammation, thrombogenicity, and atherogenic lipid profile in cigarette smokers. Circ J. 2006;70:8-13.

10. Kim BJ, Kang JG, Han JM, Kim JH, Lee SJ, Seo DC, et al. Association of self-reported and cotinineverified smoking status with incidence of metabolic syndrome in 47379 Korean adults. J Diabetes. 2019;11:402-9.

11. Kang JH, Song YM. Association between cotinine-verified smoking status and metabolic syndrome: analyses of Korean National Health and Nutrition Examination Surveys 2008-2010. Metab Syndr Relat Disord. 2015;13:140-8.

12. Kim BJ, Han JM, Kang JG, Rhee EJ, Kim BS, Kang JH. Relationship of cotinine-verified and selfreported smoking status with metabolic syndrome in 116,094 Korean adults. J Clin Lipidol. 
2017;11:638-45 e2.

13. Choi SJ, Park SH, Park HY. Increased prevalence of low high-density lipoprotein cholesterol (HDL-C) levels in Korean adults: analysis of the three Korean National Health and Nutrition Examination Surveys (KNHANES 1998-2005). Osong Public Health Res Perspect. 2011;2:94-103.

14. Connor Gorber S, Schofield-Hurwitz S, Hardt J, Levasseur G, Tremblay M. The accuracy of selfreported smoking: a systematic review of the relationship between self-reported and cotinineassessed smoking status. Nicotine Tob Res. 2009;11:12-24.

15. Benowitz NL, Jacob P, 3rd. Metabolism of nicotine to cotinine studied by a dual stable isotope method. Clin Pharmacol Ther. 1994;56:483-93.

16. Yuan C, Kosewick J, Wang S. A simple, fast, and sensitive method for the measurement of serum nicotine, cotinine, and nornicotine by LC-MS/MS. J Sep Sci. 2013;36:2394-400.

17. Kim S. Overview of cotinine cutoff values for smoking status classification. Int J Environ Res Public Health. 2016;13:1236.

18. Nam GE, Kim DH, Park YG, Han K, Choi YS, Kim SM, et al. Dose-related association between urinary cotinine-verified smoking status and dyslipidemia among Korean men: the 2008-2010 Korea National Health and Nutrition Examination Survey. Subst Use Misuse. 2014;49:1426-36.

19. Hsia SH, Pan D, Berookim P, Lee ML. A population-based, cross-sectional comparison of lipid-related indexes for symptoms of atherosclerotic disease. Am J Cardiol. 2006;98:1047-52.

20. Lemieux I, Lamarche B, Couillard C, Pascot A, Cantin B, Bergeron J, et al. Total cholesterol/HDL cholesterol ratio vs LDL cholesterol/HDL cholesterol ratio as indices of ischemic heart disease risk in men: the Quebec Cardiovascular Study. Arch Intern Med. 2001;161:2685-92.

21. Zhou M, Zhu L, Cui X, Feng L, Zhao X, He S, et al. The triglyceride to high-density lipoprotein cholesterol (TG/HDL-C) ratio as a predictor of insulin resistance but not of beta cell function in a Chinese population with different glucose tolerance status. Lipids Health Dis. 2016;15:104.

22. Maruyama C, Imamura K, Teramoto T. Assessment of LDL particle size by triglyceride/HDLcholesterol ratio in non-diabetic, healthy subjects without prominent hyperlipidemia. J Atheroscler Thromb. 2003;10:186-91.

23. Kunutsor SK, Zaccardi F, Karppi J, Kurl S, Laukkanen JA. Is high serum LDL/HDL cholesterol ratio an emerging risk factor for sudden cardiac death? Findings from the KIHD Study. J Atheroscler Thromb. 2017:24:600-8.

24. Andersson K, Arner P. Systemic nicotine stimulates human adipose tissue lipolysis through local cholinergic and catecholaminergic receptors. Int J Obes Relat Metab Disord. 2001;25:1225-32.

25. Tsiara S, Elisaf M, Mikhailidis DP. Influence of smoking on predictors of vascular disease. Angiology. 2003;54:507-30.

26. Eliasson B. Cigarette smoking and diabetes. Prog Cardiovasc Dis. 2003;45:405-13.

27. Benowitz NL, Hukkanen J, Jacob P, 3rd. Nicotine chemistry, metabolism, kinetics and biomarkers. Handb Exp Pharmacol. 2009;192;29-60. 
28. Mooradian AD, Haas MJ, Wong NC. The effect of select nutrients on serum high-density lipoprotein cholesterol and apolipoprotein A-I levels. Endocr Rev. 2006;27:2-16.

29. Kleykamp BA, Heishman SJ. The older smoker. JAMA. 2011;306:876-7.

30. Muezzinler A, Mons U, Gellert C, Schottker B, Jansen E, Kee F, et al. Smoking and all-cause mortality in older adults: results from the CHANCES Consortium. Am J Prev Med. 2015;49:e53-e63.

\section{Tables}

\section{Table 1}

Baseline characteristics of cotinine-verified smokers and non-smokers 


\section{Urinary cotinine}

\section{Parameters}

Number of participants (n)

Sex, male, \%

Age, years

Low income, \%

High education level, \%

Alcohol intake, \%

Regular exercise, \%

Lipid-lowering drug use, \%

$\mathrm{DM}, \%$

HTN, \%

Family history of dyslipidemia, \%

Waist circumference, $\mathrm{cm}$

BMI, $\mathrm{kg} / \mathrm{m}^{2}$

Serum glucose, $\mathrm{mg} / \mathrm{dL}$

Systolic blood pressure, $\mathrm{mmHg}$

Diastolic blood pressure, $\mathrm{mmHg}$

$\mathrm{TC}, \mathrm{mg} / \mathrm{dL}$

HDL-C, mg/dL

LDL-C, mg/dL

$\mathrm{TG}, \mathrm{mg} / \mathrm{dL}^{*}$

TC/HDL-C

LDL-C/HDL-C

TG/HDL-C

Daily energy intake, kcal

Daily fat intake, \%

Current smoking status
$<50 \mathrm{ng} / \mathrm{mL}$

Cotinine-verified

non-smoker

3,756

$38.4(0.8)$

$69.4 \pm 0.1$

$38(1.2)$

$31.5(1.3)$

$36.8(1)$

$41.7(1.1)$

$22.8(0.8)$

$18.5(0.8)$

$50.5(0.9)$

$2.8(0.3)$

$85.1 \pm 0.2$

$24.4 \pm 0.1$

$107.4 \pm 0.5$

$127.2 \pm 0.3$

$73.8 \pm 0.2$

$189.4 \pm 0.7$

$49 \pm 0.2$

$112.5 \pm 0.7$

$120.4(118-122.8)$

$4.05 \pm 0.02$

$2.4 \pm 0.02$

$3.2 \pm 0.1$

$1,690.7 \pm 14.7$

$14.5 \pm 0.2$

$0.2(0.1)$

Page 14/19 $\geq 50 \mathrm{ng} / \mathrm{mL}$

Cotinine-verified

smoker

593

$81.8(2)$

$<0.0001$

$68.5 \pm 0.3$

0.004

36.9 (2.3)

0.637

33.7 (2.3)

0.382

$59.1(2.2)$

$<0.0001$

$42.8(2.6)$

0.696

$12.4(1.5)$

$<0.0001$

$21.3(2)$

0.163

$51.1(2.3)$

0.821

$0.8(0.4)$

0.006

$85 \pm 0.4$

0.813

$23.4 \pm 0.1$

$<0.0001$

$111.8 \pm 1.6$

0.007

$125.4 \pm 0.9$

0.041

$72.3 \pm 0.5$

0.008

$184.8 \pm 1.8$

0.018

$45.7 \pm 0.6$

$<0.0001$

$107.1 \pm 1.7$

0.002

135.8 (128.9-143.2)

$<0.0001$

$4.3 \pm 0.1$

0.001

$2.48 \pm 0.05$

0.121

$<0.0001$

$3.9 \pm 0.1$

0.028

$1,771.6 \pm 35.1$

0.154

$15.2 \pm 0.5$

$<0.0001$ 
reported via questionnaire, \%

Urinary cotinine, $\mathrm{ng} / \mathrm{mL}^{*}$

$0.8(0.7-0.8)$

$699.2(641.5-762.1)$

$<0.0001$

Values are presented as mean \pm standard error for continuous variables or as weighted percentage (standard error) for categorical variables.

*Geometric mean (95\% confidence interval)

DM, diabetes mellitus; $\mathrm{HTN}$, hypertension; BMI, body mass index; TC, total cholesterol; HDL, highdensity lipoprotein cholesterol; LDL-C, low-density lipoprotein cholesterol; TG, triglycerides

Table 2

ORs and $95 \%$ Cls for cotinine-verified smokers (urinary cotinine $\geq 50 \mathrm{ng} / \mathrm{mL}$ ) according to various serum lipid level and serum lipid ratios 


\begin{tabular}{lllll} 
Model & $\begin{array}{l}\text { Response } \\
\text { Variable }\end{array}$ & Total & & \\
\cline { 3 - 5 } Model 1 & OR $(95 \% \mathrm{Cl})$ & $\mathrm{p}$ & $\mathrm{R}^{2}$ \\
\hline & TC Q4 & $1.07(0.83-1.39)$ & 0.589 & 0.013 \\
\hline & HDL-C Q1 & $1.67(1.32-2.12)$ & $<.0001$ & 0.038 \\
\hline & LDL-C Q4 & $0.99(0.76-1.28)$ & 0.926 & 0.006 \\
\hline & TG Q4 & $1.72(1.35-2.19)$ & $<.0001$ & 0.044 \\
\hline & TC/HDL-C ratio Q4 & $1.62(1.26-2.08)$ & 0.0002 & 0.028 \\
\hline LDL-C/HDL-C ratio Q4 & $1.38(1.07-1.76)$ & 0.011 & 0.019 \\
\hline TG/HDL-C ratio Q4 & $1.90(1.50-2.40)$ & $<.0001$ & 0.044 \\
\hline & TC Q4 & $1.04(0.78-1.38)$ & 0.791 & 0.109 \\
\hline HDL-C Q1 & $1.67(1.29-2.17)$ & 0.000 & 0.073 \\
\hline & LDL-C Q4 & $1.03(0.78-1.36)$ & 0.830 & 0.131 \\
\hline & TG Q4 & $1.49(1.14-1.96)$ & 0.004 & 0.044 \\
\hline & TC/HDL-C ratio Q4 & $1.50(1.14-1.97)$ & 0.004 & 0.103 \\
\hline & LDL-C/HDL-C ratio Q4 & $1.44(1.10-1.89)$ & 0.008 & 0.129 \\
\hline & TG/HDL-C ratio Q4 & $1.75(1.35-2.28)$ & $<.0001$ & 0.055
\end{tabular}

Data are obtained using multivariable adjusted logistic regression models and expressed as odds ratios (95\% confidence intervals).

Model 1: Adjusted for age, sex, BMI, income level, and education.

Model 2: In addition to Model 1, variables adjusted for alcohol consumption, physical activity, family history of dyslipidemia, diabetes, hypertension, use of lipid-lowering drugs, energy intake, and fat intake.

$\mathrm{OR}$, odds ratio; $\mathrm{Cl}$, confidence interval; $\mathrm{TC}$, total cholesterol; $\mathrm{HDL}-\mathrm{C}$, high-density lipoprotein cholesterol; LDL-C, low-density lipoprotein cholesterol; TG, triglycerides; Q4, highest quartile; Q1, lowest quartile

\section{Figures}


$\mathrm{n}=19,350$, assessed for eligibility,

from the KNHANES (2014-2016)

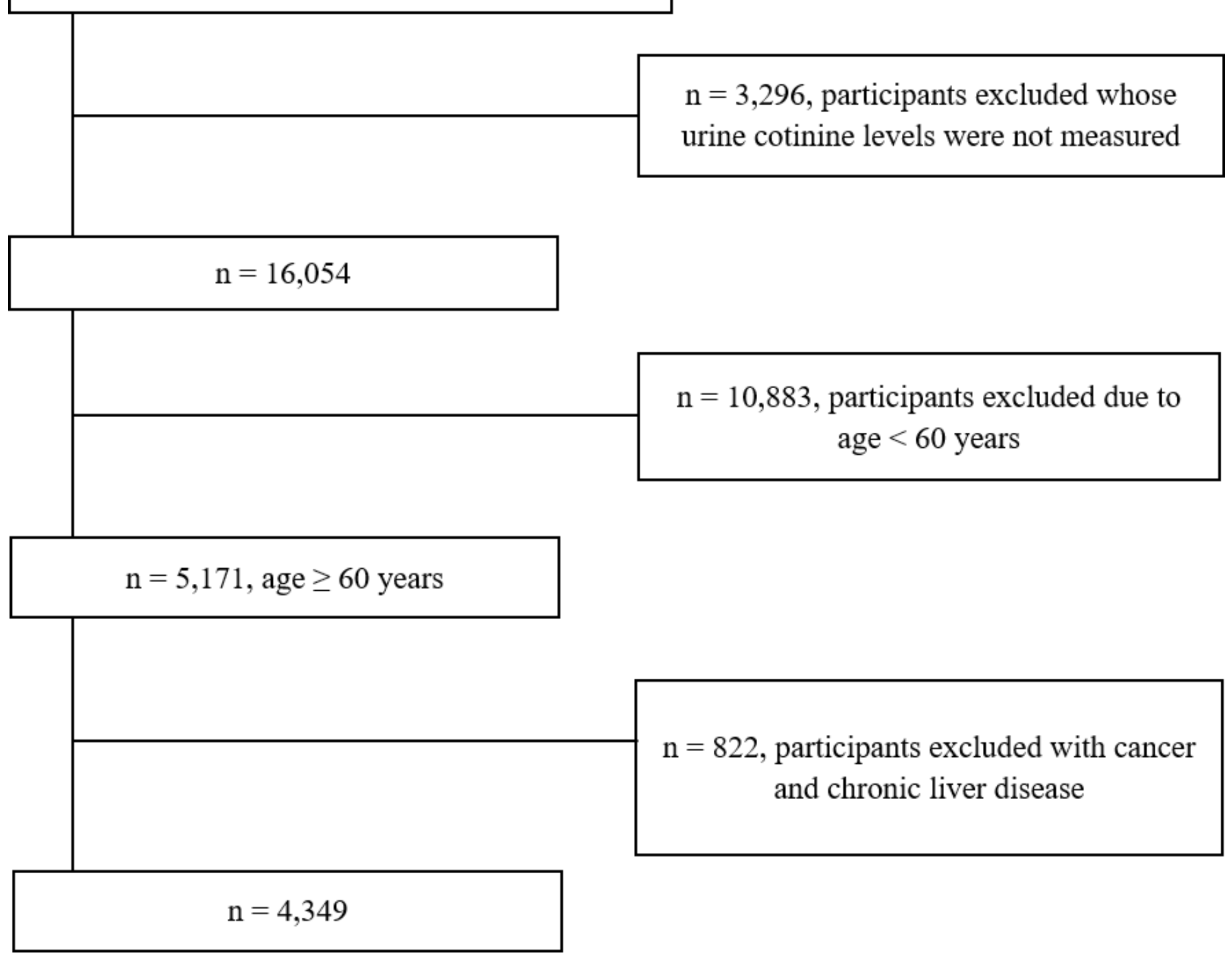

Figure 1

Flow diagram outlining the study design with the inclusion and exclusion criteria.

KNHANES, Korea National Health and Nutrition Examination Survey 


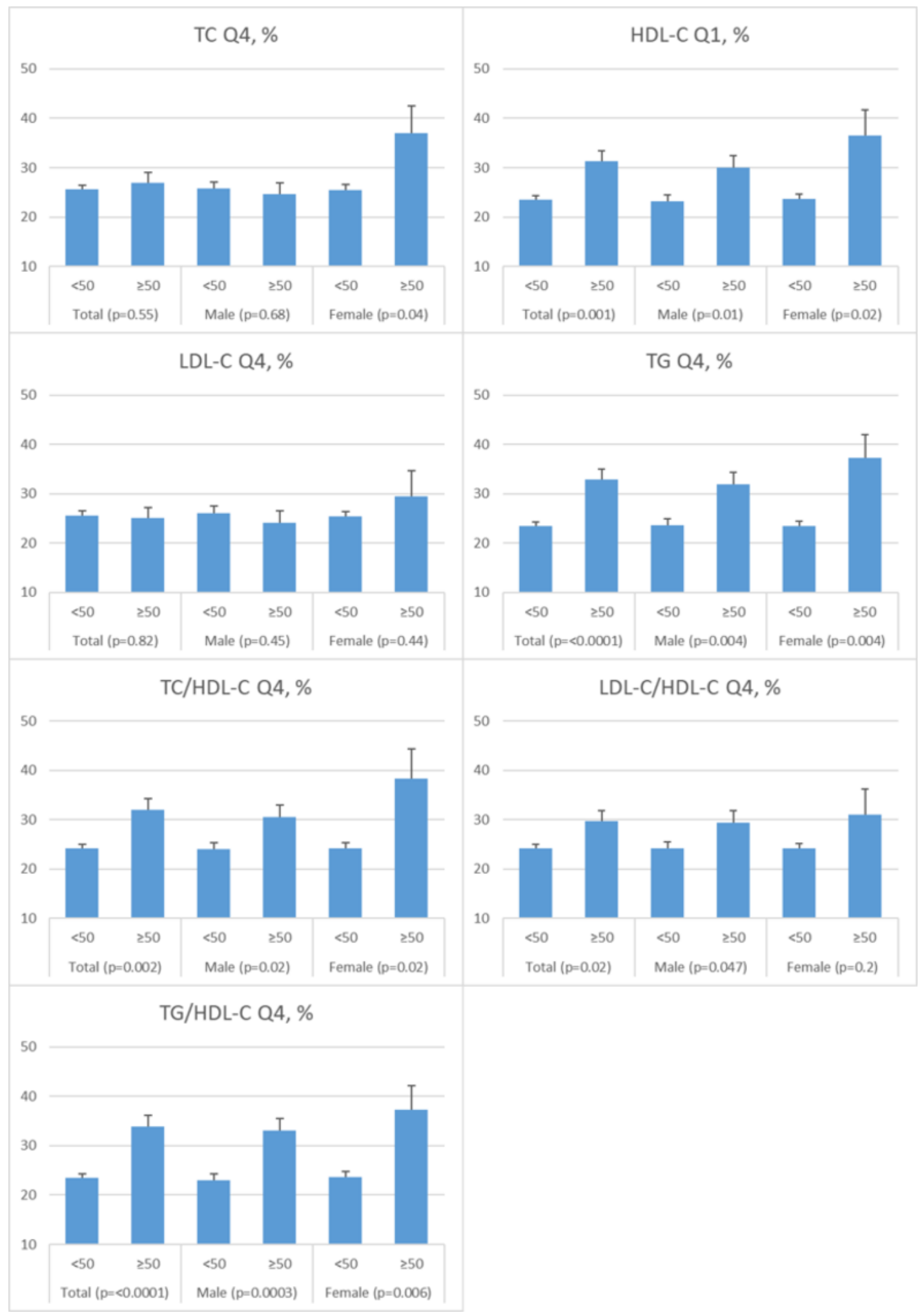

Figure 2

Distribution of unfavorable lipid parameters/ratios in cotinine-verified smokers (urinary cotinine $\geq 50$ $\mathrm{ng} / \mathrm{mL}$ ) and non-smokers.

TC, total cholesterol; HDL-C, high-density lipoprotein cholesterol; LDL-C, low-density lipoprotein cholesterol; TG, triglycerides; Q4, highest quartile; Q1, lowest quartile. 


\section{Supplementary Files}

This is a list of supplementary files associated with this preprint. Click to download.

- AdditionalFile1.pdf

- AdditionalFile2.pdf 